\title{
Mobile health (mHealth) applications with children in treatment for obesity: A randomised feasibility study
}

\author{
$\underline{\text { Sarah Browne }}^{1,2}$, Gerardine Doyle ${ }^{3}$, Tahar Kechadi ${ }^{4}$, Shane O’Donnell ${ }^{4}$ James O'Connor $^{4}$, \\ Louise Tully ${ }^{1}$, Mckenzie Dow ${ }^{5}$ and Grace O'Malley ${ }^{1,2}$ \\ ${ }^{1}$ Division of Population Health Sciences, Royal College of Surgeons in Ireland, Dublin, Ireland, \\ ${ }^{2}$ W82GO Service, Temple St. Children's University Hospital, Dublin, Ireland, \\ ${ }^{3}$ College of Business, Michael Smurfit Graduate Business School, University College Dublin, Dublin, Ireland, \\ ${ }^{4}$ Insight Centre for Data Analytics, School of Computer Science, University College Dublin, Dublin, Ireland and \\ ${ }^{5}$ School of Public Health, Physiotherapy \& Sports Science, University College Dublin, Dublin, Ireland
}

\section{Abstract}

Introduction

Smartphone mHealth apps can help children with obesity modify their rate of eating ${ }^{(1)}$ and monitor physical activity ${ }^{(2)}$. However, owing to issues with adherence, mHealth interventions require rigorous feasibility testing ${ }^{(3)}$.

\section{Aim}

To evaluate, using a randomised design, the feasibility and acceptability of a mHealth intervention to reduce rate of eating and track physical activity among children in treatment for obesity.

\section{Methods}

Children (9-16 years) with obesity (BMI $\geq 98^{\text {th }}$ centile) were recruited at a tertiary healthcare centre. The Research Ethics Committee at Temple St. Children's University Hospital granted ethical approval. Upon completing informed consent and assent, participants completed 2-week baseline testing including anthropometry, rate of eating by Mandometer ${ }^{\circledR}$ and physical activity using myBigO app. Thereafter participants were randomised to:(1)Treatment: Usual clinical care + Mandometer ${ }^{\circledR}$ training or (2)Control: Usual clinical care. Gender and age (9.0-12.9 years and 13.0-16.9 years) stratifications were applied. After a 4-week treatment period, participants repeated the 2-week testing period. Feasibility measures included fidelity with planned recruitment, randomisation, and intervention delivery and attrition. Acceptability measures included objective clinical portal engagement data and feedback from participants.

\section{Results}

Of 20 recruited, eight were randomised to intervention and 12 to control, with no significant age, gender or BMI SDS differences between groups. At baseline, 7 intervention $(87.5 \%)$ and 8 control $(66.7 \%)$ participants recorded rate of eating. Eighteen participants $(90 \%)$ registered with myBigO app, with 16 recording data successfully. Two had smartphones incompatible with myBigO $(\mathrm{n}=1$ intervention; $\mathrm{n}=1$ control) and two did not engage with myBigO app $(\mathrm{n}=1$ intervention; $\mathrm{n}=1$ control). Among 4 participants who completed Mandometer ${ }^{\circledR}$ intervention, dose received ranged from $7 \%-92 \%$ of planned meals. $37.5 \%$ intervention and $58.3 \%$ control participants completed post-intervention measures. Attrition was higher in the intervention $(n=5 ; 62.5 \%)$ than control $(n=3 ; 25 \%)$ group. Reasons cited for withdrawing included loss of interest ( $\mathrm{n}=3$ intervention), child felt overwhelmed or self-conscious ( $\mathrm{n}=2$ control), lack of time ( $\mathrm{n}=1$ intervention), behavioural issue with child ( $\mathrm{n}=1$ control), and family illness $(\mathrm{n}=1$ intervention). No significant age, gender or BMI SDS differences were observed between non-completers and completers. Participant engagement and feedback indicated mixed acceptability among this cohort.

\section{Conclusion}

Based on results, the current protocol for study design and intervention should be improved, if engagement is to be maximised.

The study is part of EU H2020 BigO Study (Big Data Against Childhood Obesity, Grant No. 727688.https://bigoprogram.eu/).

\section{Conflict of Interest}

Mandometer ${ }^{\circledR}$ equipment was supplied by the Mando Group AB, Stockholm, Sweden who are collaborative partners in the BigO Project.

\section{Reference}

1. Ford et al. (2010) BMJ 340, b5388.

2. Cooper et al. (2015) IJBNPA 12, 113

3. Hinton et al. (2018) BMC Pediatr 18, 366. 